\title{
Short Research Communication: Predicting osteopathy among patients with thalassaemia major using selected clinical and biochemical information
}

\author{
Jayasinghe SS ${ }^{1}$, Lekamwasam S ${ }^{2}$, De Silva $\mathrm{DCL}^{3}$, Amarasena TSD ${ }^{3}$, Subasinghe L ${ }^{1}$, Purasinghe ${ }^{4}$ \\ Departments of ${ }^{1}$ Pharmacology, ${ }^{2}$ Medicine, ${ }^{3}$ Paediatrics and ${ }^{4}$ Allied Health Science Unit, Faculty of Medicine, \\ University of Ruhuna, Galle, Sri Lanka
}

Correspondence: Dr.S.S. Jayasinghe; (sudheerasj@yahoo.com)

\begin{abstract}
Objectives: This study assessed the prevalence and surrogate makers of osteopathy among patients with thalassemia major.

Methods: Bone mineral densities (BMDs) were measured by dual-energy x-ray absorptiometry of the lumbar spine and total hip in 34 patients with thalassemia major. Postprandial glucose, serum ferritin, vitamin $\mathrm{D}_{3}$ levels were also measured. Osteopathy was defined as the presence of either a low-energy fracture or BMD of at least one site; total spine or total hip, 2 standard deviations below the mean of age and sex-matched normal population (Z-score).

Results: In 34 patients recruited median (inter-quartile range) disease period was 8.2 (5.6 - 12.5) years. Two patients had suffered low energy fractures previously while 12 patients $(14 / 34,41 \%)$ had osteopathy either in the total spine or total hip. Patients with osteopathy were heavier $(29.7 \mathrm{~kg}$ vs $22.8 \mathrm{~kg}, \mathrm{P} 0.045)$ and taller (130 cm vs $122 \mathrm{~cm}, \mathrm{P}$ 0.028) when compared to thalassaemics with no osteopathy. The liver span, number of blood transfusions, prevalence of splenomegaly, and body mass index were not significantly different between the patients with and without osteopathy. Postprandial glucose, serum ferritin and vitamin D were similar between the patients with and without osteopathy. When these variables were included in a logistic regression model, no significant clinical predictors were recognized.
\end{abstract}

Conclusions: Osteopathy is a common complication of thalassaemia major and no clinical or biochemical predictors of osteopathy were recognized.

Key words: Beta-thalassaemia, Bone density, Bone disease, Ferritin, Osteoporosis

\section{Introduction}

Despite the improvement in life expectancy over the last decade, the long-term complications of thalassaemics are still common (1). Osteopathy is a well known complication of thalassaemia major (TM) and is associated with increased morbidity (2). These patients are susceptible to bone pains, spontaneous fractures, necrosis of the femoral head, vertebral fractures and cord compression (3).
In Sri Lanka, patients with TM are not routinely screened for the presence of osteopathy. This may be a result of low awareness of this complication or restricted availability of dual-energy $\mathrm{x}$-ray absorptiometry (DXA) which is required to make the diagnosis. Furthermore, studies on the prevalence of osteopathy among patients with TM in Asian countries are sparse.

Apart from the prevalence, it is equally important to know the surrogate markers or clinical predictors of 
osteopathy among thalassaemics. These surrogate markers would help in screening patients with TM before referring them for DXA evaluation.

\section{Methods}

This cross-sectional study was approved by the local Ethics Committee. Consecutive patients with TM diagnosed and managed by either a specialist physician or paediatrician were recruited after obtaining informed written consent from parents / patients and assent from the patients who were below the age of 16 years. All patients underwent a detailed physical examination. Their weight and standing height were measured (Weight Master International, model No BW-110H) while wearing light cloths and without footwear. All patients underwent bone mineral density (BMD) assessment using Hologic Discovery scanner (Hologic Inc, Bedford, MA, USA). BMDs of total spine from L1 to L4 and left proximal femur (total hip and femoral neck) were estimated. In-vivo precision of BMD estimations of the same machine has been published elsewhere (4).

Z-scores (the difference between patient's BMD and mean BMD of age and sex-matched normal population, expressed as a function of same population standard deviation) were calculated for each BMD measurement using the reference data provided by the DXA manufacturer. Patients who had Z-score equal or less than - 2.00, either in the total spine or proximal femur were considered to have osteopathy. In addition, patients with low energy fractures were also considered to have osteopathy, irrespective of their BMDs.

One milliliter of blood was drawn two hours following lunch for the estimation of postprandial blood glucose (PPBG) (Diagnostica - Merk). Vitamin D3 (IDS OCTEIA 25-hydroxyvitamin D) and serum ferritin were estimated between two blood transfusions (IDS OCTEIA serum ferritin).

Differences between patients with and without osteopathy were compared by unpaired t-test. Logistic regression model was used to find out the determinants of osteopathy. $\mathrm{P}$ value was adjusted by the Bonferroni method to accommodate multiple comparisons.

\section{Results}

Out of 40 TM patients admitted to Teaching Hospital, Karapitiya during this period, 34 who were above the age of three years were recruited. Their age ranged from 3.5 to 25 years (table 1). Sixteen patients were above the age of 10 years while 18 were males. Median (Inter-Quartile Range) number of blood transfusions patients received was 60 (36 - 96). Ten patients were on intravenous desferrioxamine (100 mg/kg body weight) while four patients were on subcutaneous desferrioxamine. The rest of the patients were on both intravenous and subcutaneous desferrioxamine. Twelve patients had undergone splenectomy while in 15 patients spleen was not palpable. The liver was enlarged in all patients and mean (SD) of liver span was $14.5(3.2) \mathrm{cm}$.

Table 1: Descriptive data of patients with thalassemia major

\begin{tabular}{ll}
\hline Characteristic & Mean (SD) \\
\hline Age (years) & $12(6)$ \\
Height $(\mathrm{cm})$ & $127.9(16.5)$ \\
Weight $(\mathrm{Kg})$ & $25.6(9.4)$ \\
BMI $\left(\mathrm{Kg} / \mathrm{m}^{2}\right)$ & $15.0(1.9)$ \\
Disease duration (months) & $116(69)$ \\
\hline
\end{tabular}

Two patients had suffered low energy fractures already and further 12 had Z-score equal or below 2.00 in at least one of the sites investigated giving $41 \%$ (14 of 34) prevalence of osteopathy among this group. Mean BMDs and Z-scores of the two skeletal sites are shown in table 2 . These 14 patients with osteopathy were heavier ( $29.7 \mathrm{vs} 22.8 \mathrm{~kg}, \mathrm{P} 0.045)$ and taller (130 vs $122 \mathrm{~cm}, \mathrm{P}$ 0.028) when compared to thalassaemics without osteopathy. When analyzed based on age, the prevalence of osteopathy among those below 10 years was $27.7 \%$ (5/18). Among patients aged 10 years or more the prevalence was $50 \%(8 / 16)$.

Serum ferritin level exceeded $800 \mathrm{ng} / \mathrm{mL}$ in $11 / 34$ $(32 \%)$ patients. The mean (SD) of serum ferritin level of the rest of the patients are shown in table 2. According to the Lips classification (5), 22 patients had normal vitamin D3 level. Six patients had mild 
vitamin D3 deficiency $(25-50 \mathrm{nmol} / \mathrm{L})$ while three patients showed moderate deficiency (12.5 - 25 $\mathrm{nmol} / \mathrm{L})$. None of the patients had severe deficiency of vitamin D3. We were unable to collect blood samples for Vitamin D3 estimation in three patients. PPBG was more than $11.1 \mathrm{mmol} / \mathrm{L}$ in one patient while others had normal PPBS level. The liver span, number of blood transfusions, prevalence of splenomegaly (those who had spleen removed or palpable), BMI, serum ferritin, vitamin $\mathrm{D}_{3}$ level and PPBG were not significantly different between the patients with and without osteopathy. Furthermore, when these variables were included in a logistic regression model, no significant predictors were found.

Table 2: The mean (SD) of post-prandial blood glucose, serum vitamin D3 and BMD with Z score

\begin{tabular}{ll}
\hline \multicolumn{1}{l}{ Investigation } & Mean (SD) \\
\hline $\begin{array}{l}\text { Post-prandial blood glucose } \\
(\mathrm{mg} / \mathrm{dL})\end{array}$ & $105(29)$ \\
Serum ferritin $(\mathrm{ng} / \mathrm{mL})$ & $474(140)$ \\
Vitamin D3 $(\mathrm{nmol} / \mathrm{L})$ & $95(71)$ \\
Lumbar spine & \\
BMD $\left(\mathrm{g} / \mathrm{cm}^{2}\right)$ & $0.583(0.136)$ \\
Z-score & $-1.58(1.45)$ \\
Total hip & $0.767(0.171)$ \\
BMD $\left(\mathrm{g} / \mathrm{cm}^{2}\right)$ & $-0.33(1.53)$ \\
Z-score & \\
Femoral neck & $0.611(0.134)$ \\
BMD $\left(\mathrm{g} / \mathrm{cm}^{2}\right)$ & $-1.35(1.19)$ \\
Z-score & \\
Trochanter & $0.657(0.112)$ \\
BMD $\left(\mathrm{g} / \mathrm{cm}^{2}\right)$ & $0.1(2.6)$ \\
Z score &
\end{tabular}

\section{Discussion}

We found an overall $41 \%$ prevalence of osteopathy among this group of patients based on the criteria we used. No anthropometric, clinical or biochemical surrogates or determinants of osteopathy were found in this analysis.
Prevalence of osteopathy reported among thalassaemics varies widely. Direct comparisons of these studies are not appropriate due to the differences in age composition, severity of the disease and criteria used to define osteopathy. While we considered $Z$-score equal or -2.00 cut-off value to define osteopathy as recommended by the International Society of Clinical Densitometrists, others $(2,6)$ have used other Z-score thresholds. Jensen CE et al. (6) reported $96.3 \%$ an overall prevalence of osteopathy while Shamshirsaz AA, et al. (2) reported $90 \%$ prevalence. The different Z-score cut off value they used would have contributed to the high prevalence observed in their analyses. In 2010, Merchant et al reported 81\% prevalence of osteopathy among Indian thalassaemics aged between 10-25 years (7). This is higher than $50 \%$ prevalence we observed among patients above 10 years in our sample. Patients in Merchant's analysis, however, had a higher prevalence of hypovitaminosis D (62\%) and this may have contributed to the higher prevalence of osteopathy they observed.

BMD is positively related to weight and height in normal children (8) but this association was not seen among our study subjects. Vitamin D which is a well known determinant of BMD in other age groups $(9,10)$, was not a significant predictor of osteopathy among our subjects. In support of our observations, Rioja L, et al. (1990) could not find significant associations between bone disease and vitamin D deficiency or iron overload in thalassemia major patients (11). Furthermore, Merchant et al, found no difference in serum ferritin, vitamin D and PTH between Indian thalassaemics with and without osteopathy (7).

Our study has several limitations. Small sample size may have contributed to some inconclusive or negative results. Furthermore, the study sample was heterogeneous with regards to age and the duration of the illness, limiting the external validity of results. Lack of bone markers data did not allow us to study the mechanism of bone loss among thalassaemics. Our data however, can be used as a platform for future research on this subject and also to convince clinicians regarding the need of DXA assessment in all thalassaemics. 


\section{Conclusion}

Osteopathy is a common complication of TM and no clinical or biochemical predictors of osteopathy could be recognized in these patients. Until reliable surrogate markers of thalassaemic osteopathy are recognized, all patients with TM should have DXA evaluation to assess their fracture risk.

\section{References}

1. Olivieri N. F, Brittenham G. M, McLaren C. E, Templeton D. M, Cameron R. G, McClelland R. A, Burt A. D, Fleming $\mathrm{K}$. A: Long term safety and effectiveness of iron-chelation therapy with deferiprone for thalassemia major. The New England Journal of Medicine 1998; 339: 417-23.

2. Shamshirsaz A.A, Bekheirnia M.R, Kamgar M, Pourzahedgilani N, Bouzari N, Habibzadeh M, Hashemi R, Shamshirsaz A. A, Aghakhani S, Homayoun H, Larijani B: Metabolic and endocrinologic complications in betathalassaemia major: a multicenter study in Tehran. BMC Endocr Disord 2003;3:4.

3. De Sanctis V, Angastiniotis M, Eleftheriou A: Pediatric endocrinology reviews. Diabetes, nutrition, metabolism. Bone disease and endocrine complications in thalassaemia. Forward. Pediatr Endocrinol Rev 2008; 6(Suppl 1): 71-2.

4. Lekamwasam S, Rodrigo M, Arachchi WK, Munidasa D: Measurement of spinal bone mineral density on a Hologic Discovery DXA scanner with and without leg elevation. JClin Densitom 2007; 10: 170-3.
5. Lips P: Vitamin D deficiency and secondary hyperparathyroidism in the elderly: consequences for bone loss and fractures and therapeutic implications. Endocr Rev 2001; 22: 477-501.

6. Jensen C.E, Tuck S.M, Agnew J.E, Koneru S, Morris R. W, Yardumian A, Prescott E, Hoffbrand A.V, Wonke B: High prevalence of low bone mass in thalassaemia major. British Journal of Haematology 1998; 103: 911-5.

7. Merchant R, Udani A, Puri V, D'Cruz V, Patkar D, Karkera A: Evaluation of osteopathy in thalassaemia by bone mineral densitometry and biochemical indices. Indian $J$ Pediatr 2010; 77: 987-91.

8. Lee S. H, Desai S. S, Shetty G, Song H. R, Lee S. H, Hur C. Y, Lee J. C: Bone mineral density of proximal femur and spine in Korean children between 2 and 18 years of age. JBone Miner Metab 2007; 25: 423-30.

9. NIH Consensus Development Panel on Osteoporosis Prevention, Diagnosis, and Therapy. Osteoporosis prevention, diagnosis, and therapy. JAMA 2001; 285: 785 95.

10. Dawson-Hughes B: Racial/ethnic considerations in making recommendations for vitamin D for adult and elderly men and women. Am JClin Nutr 2004; 80: 1763S-6S.

11. Rioja L, Girot R, Garabedian M, Cournot-Witmer G: Bone disease in children with homozygous beta-thalassaemia. Bone Miner 1990; 8: 69-86. 